\title{
Detection of Rift Valley Fever Virus Interepidemic Activity in Lower Moshi area of Kilimanjaro Region, North Eastern Tanzania: A Community Survey
}

Jaffu Chilongola ( $\nabla$ j.chilongola@kcri.ac.tz)

Kilimanjaro Christian Medical University College

Medard Kumalija

Nelson Mandela African Institution of Science and Technology

Rule Budodo

Kilimanjaro Clinical Research Institute

Pius Horumpende

Lugalo Military College of Medical Sciences

Sixbert Mkumbaye

Kilimanjaro Clinical Research Institute

John-Mary Vianney

Nelson Mandela African Institution of Science and Technology

Richard Mwakapuja

Tanzania Veterinary Laboratory Agency, Kibaha

Blandina Mmmbaga

Kilimanjaro Clinical Research Institute

\section{Research Article}

Keywords: Epidemiology, seroprevalence, inter-epidemic, Rift Valley Fever, Tanzania

Posted Date: January 22nd, 2021

DOI: https://doi.org/10.21203/rs.3.rs-143963/v1

License: (c) (i) This work is licensed under a Creative Commons Attribution 4.0 International License. Read Full License 


\section{Abstract}

Background: Rift Valley fever virus (RVFV) is a zoonotic arbovirus of public health impact infecting livestock, wildlife, and humans mainly in Africa and other parts of the world. Despite its public health importance, mechanisms of RVFV maintenance during inter-epidemic (IEPS) periods and potentially spread to new areas remain unclear.We aimed to comparatively examine exposure to RVFV and RVFV infection among humans, goats and mosquitoes in an agro-pastoral community in Lower Moshi area of Moshi rural district.

Results:Results show that the male gender was related to RVFV seropositivity $(\chi 2=5.351 ; p=0.030)$. Being 50 years and above was related to seropositivity $(\chi 2=14.430 ; p=0.006)$ whereas bed net use, larger numbers of persons living in the same house ( $>7$ persons) and RVFV seropositivity in goats were related to higher seropositivity to RVFV among humans $(\chi 2=6.003 ; p=0.021, \chi 2=23.213 ; p=0.000$ and $27.053 ; p=0.000)$, respectively.RVFV antibody concentrations were only marginally higher in humans without statistically significant difference $[\mathrm{t}(112)=0.526 ; \mathrm{p}=0.60)]$. By the use of RT-qPCR, goats exhibited the highest RVFV infection rate of $4.1 \%$, followed by humans (2.6\%), Aedes spp(2.3\%), and Culex spp(1.5\%).

Conclusions: In the absence of RVFV infection data in areas nearby the study site, our findings suggest Lower Moshi area as a potential hotspot for RVF, posing the danger of being a source of RVFV spread to other areas. Goats had the highest infection rate, suggesting goats as important hosts in the virus maintenance during IEPs. We recommend the design and implementation of strategies that will warrant effective active surveillance of RVF through the identification of RVF hotspots for targeted control of RVF.

\section{Background}

Rift Valley fever virus (RVFV) is a zoonotic arbovirus affecting livestock and humans mainly in Africa and the Arabian Peninsula [1-4] although recent reports indicate the presence of RVF in other parts of the world[5]. According to the World Health Organization (WHO), Rift Valley fever (RVF) is a priority disease due to its considerable public health impact in areas where it occurs and the inadequate interventions to control it [6]. It is also considered an important threat to agriculture in African countries including Tanzania [7-9]. Transmission of RVFV to animals is mainly through bites by infected Aedes and Culex mosquitoes, whereas human transmission largely through direct contact with tissues of RVFV-infected animals[10].

It has been previously suggested that maintenance of the virus in animals during inter-epidemic (IEPS) periods and potentially spread to new areas through animal movement. Disease pathology and endemic maintenance within mammalian hosts have been reviewed [11,12]. Although the transmission of RVFV by mosquito vectors to animals and humans has been described, less is known about the role of animals, humans, and vector mosquitoes in maintaining the virus during IEPS. The maintenance mechanisms during IEPS become interesting due to the absence of a clear understanding of where the virus hides during the "silent" periods. Some explanations have been made regarding the possible mechanisms by which the virus is maintained during IEPS. Previous work has documented low-levels of RVFV exposure in northern, central, eastern, and southern Tanzania as a key mechanism of virus maintenance [13, 14]. Most people infected by RVFV remain asymptomatic although a small percentage present with clinical disease. Other reports have 
hypothesized critical mechanisms for survival of RVFV during long inter-epizootic periods as vertical transmission through mosquito eggs to mosquito offspring[15-17].

Maintenance of RVFV depend on the presence of competent vectors and hosts but must coincide with multiple factors such as sufficient livestock density, rainfall providing vector breeding sites, and temperatures that support vector development and pathogen replication [18], but differential exposure of RVFV in high-risk agropastoral communities in Northern Tanzania has not been examined. We aimed to comparatively examine exposure to RVFV and RVFV infection among humans, goats and mosquitoes in an agropastoral community in Lower Moshi area of Moshi rural district.

\section{Methods}

\section{Study Design and Site}

A community-based, cross-sectional survey was conducted in three villages of lower Moshi in Moshi district, Kilimanjaro region of Tanzania. Data were collected between March and June 2020 involving 3 villages, namely Mikocheni, Chemchem, and Arusha Chini. Lower Moshi is located on the southern foothills of Mount Kilimanjaro (Figure 1). On the west, Lower Moshi is bordered by the Kikuletwa River, Hai District, and Manyara Region. To the east Lower Moshi borders Mwanga district. Lower Moshi elevation ranges between 700 and 800 m above sea level. The main Rift Valley Fever vectors in this area are Culex spp, Mansoniaspp, Anopheles spp, and Aedes spp[19]. Numerous water streams cross the area and they form the irrigation channels for rice and sugar cane. The rice irrigation schemes have structured and unstructured canal networks; covering an area of about 1,100 hectares. During the rainy season, temporary pools that serve as malaria vector breeding sites are formed. Their persistence beyond the rains contributes to further malaria transmission. The area has two rainy seasons; the long rains which run from March to May and the short rainy season from November to December. The average annual rainfall is about $900 \mathrm{~mm}$ per year[20].

\section{Participants and sample collection}

Participants in this study were males and females aged between 10 and 70 years, who were either smallholder crop farmers or livestock keepers and willing to participate in this study. Animal sampling was carried out by animal health experts from the Tanzania Veterinary Laboratory Agency (TVLA). Up to 15 goats were selected from each herd by systematic sampling technique where every $3^{\text {rd }}$ and $5^{\text {th }}$ animal was included depending on the size of the herd. Selected animals were manually restrained and $3 \mathrm{ml}$ of blood collected through jugular venipuncture using a vacutainer needle. Human blood sampling was done by expert phlebotomists from the Kilimanjaro Christian Medical Center (KCMC). Three milliliters of blood were collected from the median cubital vein by venipuncture. Each sample from both animals and humans was divided into two aliquots of $1.5 \mathrm{ml}$ each and placed into plain and EDTA vacutainer tubes, respectively. To each sample in an EDTA tube, $4.5 \mathrm{ml}$ of Tri Reagent (Zymo Research, Irvine, CA, U.S.A.) were added. The mixture was gently mixed by shaking for 1 minute and immediately shipped to the KCRI biotechnology laboratory at $4^{\circ} \mathrm{C}$, for analyses. Demographic data from participants were collected using electronic forms designed using Open Data Kit (ODK)tools (https://opendatakit.org/) deployed in Android tablets. 


\section{Mosquito trapping}

BG Sentinel trap (BGS) (Biogents AG, Regensburg, Germany) to target outdoor host-seeking adult mosquitoesparticularlyAedes spp, Ochlerotatusspp, Culex spp, Mansoniaspp, and Anopheles spp[21]. BGS traps were used in combination with the BGS-Lure, a dispenser that releases emanations such as those found on human skin (lactic acid, ammonia, and caproic acid)[22]. The BGS-Trap, developed by BioGents $\mathrm{GmbH}$ (Regensburg, Germany), consists of an easy to transport, collapsible white bucket with white gauze covering its opening. In the middle of the gauze cover, there is a black tube through which a downflow is created by $12 \mathrm{~V} \mathrm{DC}$ fan that causes any mosquito in the vicinity of the opening tube sucked into a catch bag[22]. Mosquitoes were immediately morphologically identified in the field and consequently sorted according to species, sex, and whether fed or unfed.

\section{Laboratory procedures}

\section{RVFV competitive ELISA (cELISA)}

Serumwas extracted from the plain vacutainer tubes at the end of eachday by centrifugation of clotted blood at3000 rpm for $5 \mathrm{~min}$. An extracted serum sample was then transferred into $2 \mathrm{ml}$ sterile cryovials using a sterile Pasteur pipette. All serum samples were tested for the presence of antibodies against RVFV using a competitive ELISA (cELISA)using the ID Screen RVFCompetition Multi-Species kit (ID-vet, Grables, France), which detects both IgG and IgM antibodies directed against the RVFV nucleoprotein (NP). Validation tests for the test kit have shown a sensitivity of between 91 and $100 \%$ and a specificity of $100 \%$. The cELISA was performed according to the instructions of the manufacturer and as described previously [23, 24]. To control the validity of each plate, the mean value of the two negative controls $\left(O D_{N C}\right)$ was computed whereby a plate was considered valid if the $O D_{N C}$ was $>0.7$. For a valid plate, the mean value of the two positive controls divided by $O D_{N C}$ had to be $<0.3$. For each sample, the competition percentage was calculated by dividing $\left.O D_{\text {sample }} / O D_{N C}\right) \times 100$. If the value was $\leq 0.4$, the sample was considered positive while a value $>0.5$ was considered negative. Only samples that tested positive for cELISA were subjected to RT-qPCR for RVFV detection.

\section{Ribonucleic acid (RNA) isolation, purification, and real-time PCR amplification.}

For detection of RVFV RNA in humans and goats, RNA was extracted from Trizol archived blood in EDTA tubes using DirectZol miniprep kit (Zymo Research, Irvine, CA, U.S.A.) by using the Boom method. To isolate RVFV RNA from mosquitoes, pools of 10-50 unfed monospecific female mosquitoes were placed in cryovials and transferred into Lysing Matrix, impact-resistant tubes containing $1.4 \mathrm{~mm}$ ceramic beads (MP Biomedicals, CA, USA). Samples were disrupted by bead beating at $10,000 \mathrm{xg}$ for $1 \mathrm{~min}$, spun at $1000 \mathrm{~g}$ for $10 \mathrm{~min}$ at $4^{\circ} \mathrm{C}$. The supernatant was transferred into labeled RNase-free tubes. Purification procedures were done using Direct-zol ${ }^{\text {TM }}$ RNA miniprep kit (Irvine, CA, U.S.A) following the manufacturer's instructions.

For both human/goat and mosquito samples, RNA concentration and quality check were performed using NanoDrop ${ }^{\mathrm{TM}} 2000$ Spectrophotometer (Thermo Scientific, NY, USA) before storage at $-80^{\circ} \mathrm{C}$. RVFV RNA was 
detected using TaqMan probe-based one-step RT-qPCR targeting the RVFV Gn gene as described by Gudo and colleagues [2] using Applied Biosystems ViiA7 PCR platform, Thermo Scientific, NY, USA).

\section{Nature of data and data Analysis}

Data analysis was performed using IBM SPSS v.26 (IBM® Corp., Armonk, NY, USA). Descriptive data were presented as frequencies and percentages, means, and medians. Categorical data were reported as a tabulation of proportions and compared between humans and goats. Chi-squared statistic $\left(\chi^{2}\right)$ was used to examine associations between seropositivity to RVFV and RVFV infection in both humans and goats. Mean $\lg \mathrm{M}$ and IgG concentrations were compared between humans and goats by paired t-test. Percent positivity to RVFV infection in goats, humans, and mosquitoes were reported as histograms.

\section{Ethical issues}

This study obtained approval by the Kilimanjaro Christian Medical University College (KCMUCo) Research and Ethics Committee (CRERC) with approval certificate \#2419. This study was also approved by the Kilimanjaro Regional and District Administrative Secretaries, District Medical and Veterinary Officers, and local village and ward executive officers of respective villages. Before commencement of sample collection, written informed consent was obtained from all study participants aged 18 years and above by signing consent forms whereas parents and/or legal guardians of participants under 18 years and participants who could not read or write consented on behalf. All authors hereby confirm that all procedures in this study were approved by CRERC and were performed in accordance with the ethical standards as laid down in the 1964 Declaration of Helsinki. Authors also confirm that all procedures that involved animals in this study were conducted in compliance with the ARRIVE guidelines.

\section{Results}

\section{Demographic Characteristics of Human Participants}

A total of 266 human participants were enrolled in the study. Of the participants, more than half (56.4\%) were females. The median age (interquartile range) of participants was $45(30-55)$. The majority of participants $(74.4 \%)$ came from households with more than 4 persons in the same house. With regards to the participant's education, $63.2 \%$ of participants had attained primary school education. Most participants (72.9\%) kept livestock (cattle, sheep, goats, and/or chicken). Three quarters (75.2\%) of the participants reported having used an insect side treated bed-net the night before the interview. (Table 1) 
Table 1

Demographic Characteristics of Participants

\begin{tabular}{|lll|}
\hline Characteristics & $\mathbf{n}$ & $\%$ \\
\hline Age group (years) & & \\
\hline$\leq 20$ & 28 & 10.5 \\
\hline $21-50$ & 140 & 52.7 \\
\hline$>50$ & 98 & 36.8 \\
\hline (Median, IQR) years & $45(30-55)$ & \\
\hline Sex & & \\
\hline Male & 116 & 43.6 \\
\hline Female & 150 & 56.4 \\
\hline Individuals living in a household & & \\
\hline$<4$ & 68 & 25.6 \\
\hline$\geq 4$ & 198 & 74.4 \\
\hline Highest education & & \\
\hline No formal & 51 & 19.2 \\
\hline Primary & 168 & 63.2 \\
\hline Tertiary & 47 & 17.7 \\
\hline Type of animals kept by the participant & & \\
\hline Animal Keeping & 194 & \\
\hline None & 72.9 \\
\hline Bed-net use* & & \\
\hline Yes & & \\
\hline No & & \\
\hline IQR, Interquartile Range; & & \\
\hline
\end{tabular}

\section{Factors Associated With Rvfv Seropositivity In Humans And Goats}

Human RVFV seropositivity was analyzed for any associations with participant age, bed net use within the last 24 hours, positivity for RVFV infection, number of persons living under the same roof, recent travel outside the study site, highest education of the participant, and RVFV infection and seropositivity in goats, 
results are presented in Table 2 . Results show that the male gender was significantly more related to RVFV seropositivity $\left(X^{2}=5.351 ; p=0.030\right)$. Likewise, participants aged 50 years and above were more seropositive as compared to their younger counterparts $\left(X^{2}=14.430 ; p=0.006\right)$. Bed net use, larger numbers of persons living in the same house ( $>7$ persons), and RVFV seropositivity in goats were related to higher seropositivity to $\operatorname{RVFV}\left(X^{2}=6.003 ; p=0.021, \chi^{2}=23.213 ; p=0.000\right.$ and 27.053; $\left.p=0.000\right)$, respectively (Table 2). Among the selected factors analyzed for possible association with IgM/lgG RVFV seropositivity in goats, only IgM/IgG RVFV seropositivity in humans had a significant relationship $\left(\chi^{2}=27.053 ; p=0.000\right)$ (Table 3$)$.

\subsection{Comparison of mean IgM/lgG concentrations in humans and goats}

When mean concentrations of antibodies to the RVF virus were compared between goats and humans, it was observed that RVFV antibody concentrations were only marginally higher in humans without statistically significant difference $[\mathrm{t}(112)=0.526 ; \mathrm{p}=0.60$ ) (Table 4). Percentages of RVFV seropositive humans and goats as well as PCR results for viral infections were determined (Fig. 2). Compared to humans, goats were more seropositive to RVFV (23.3\% seropositive goats against $13.2 \%$ seropositive humans). 
Table 2

Factors associated with RVFV seropositivity in humans

\begin{tabular}{|c|c|c|c|c|}
\hline Variable & Level & $\begin{array}{l}\text { Negative, } \\
\mathrm{n}(\%)\end{array}$ & $\begin{array}{l}\text { Positive, } \\
\text { n(\%) }\end{array}$ & $\chi^{2}(p)$ \\
\hline \multirow[t]{2}{*}{ Sex } & Male & $47(71.2)$ & $19(28.8)$ & \\
\hline & Female & $90(85.7)$ & $15(14.3)$ & $5.351(0.030)$ \\
\hline \multirow[t]{5}{*}{ Age Group } & $11-20$ & $18(94.7)$ & $1(5.3)$ & \\
\hline & $21-30$ & $26(92.9)$ & $2(7.1)$ & \\
\hline & $31-40$ & $23(88.5)$ & $3(11.5)$ & \\
\hline & $41-50$ & $19(82.6)$ & $4(17.4)$ & \\
\hline & $>50$ & $51(67.1)$ & $25(32.9)$ & $14.430(0.006)$ \\
\hline \multirow[t]{2}{*}{ Human RVFV PCR } & Positive & $0(0)$ & $7(100)$ & \\
\hline & Negative & $1(3.4)$ & $28(96.6)$ & $0.248(1.000)$ \\
\hline \multirow[t]{2}{*}{ Bed-Net Use } & Yes & $24(64.9)$ & $13(35.1)$ & \\
\hline & No & $110(83.3)$ & $22(16.7)$ & $6.003(0.021)$ \\
\hline \multirow[t]{3}{*}{ Number of persons in a $\mathrm{HH}$} & $1-3$ & $45(90)$ & $5(10)$ & \\
\hline & $4-6$ & $74(85.1)$ & $13(14.9)$ & \\
\hline & $7+$ & $17(50)$ & $17(50)$ & $23.213(0.000)$ \\
\hline \multirow[t]{2}{*}{ Travel outside site } & Yes & $48(82.8)$ & $10(17.2)$ & \\
\hline & No & $89(78.1)$ & $25(21.9)$ & $0.521(0.551)$ \\
\hline \multirow[t]{3}{*}{ Destination } & Urban & $22(88)$ & $3(12)$ & \\
\hline & Peri-urban & $10(90.9)$ & $1(9.1)$ & \\
\hline & Rural & $16(72.7)$ & $6(27.3)$ & $2.545(0.346)$ \\
\hline \multirow[t]{3}{*}{ Highest Education } & $\begin{array}{l}\text { No Formal } \\
\text { Education }\end{array}$ & $34(81)$ & $8(19.8)$ & \\
\hline & Primary & $91(78.4)$ & $25(21.6)$ & \\
\hline & tertiary & $12(85.7)$ & $2(14.3)$ & $0.465(0.830)$ \\
\hline \multirow[t]{2}{*}{ RVFV Infection in goats } & Yes & $1(50)$ & $8(80)$ & \\
\hline & No & $1(50)$ & $2(20)$ & $0.800(1.000)$ \\
\hline \multirow{2}{*}{$\begin{array}{l}\text { RVFV seropositivity in } \\
\text { goats }\end{array}$} & Yes & $11(9.4)$ & $24(43.6)$ & \\
\hline & No & $106(90.6)$ & $31(56.4)$ & $27.053(0.000)$ \\
\hline
\end{tabular}


Table 3

Factors associated with RVFV seropositivity in goats

\begin{tabular}{|lllll|}
\hline Variable & Level & Negative, $n(\%)$ & Positive, $n(\%)$ & $\chi^{2}(\mathbf{p})$ \\
\hline RVFV infection in goats & Positive & $3(75.0)$ & $8(89.9)$ & \\
\hline Negative & $1(25.0)$ & $1(11.1)$ & $0.410(1.000)$ \\
\hline & $<20$ & $19(39.6)$ & $11(39.3)$ & \\
\hline & $20-50$ & $16(33.3)$ & $8(28.6)$ & \\
\hline IgM/IgG seropositivity in Humans & Positive & $11(9.4)$ & $24(43.6)$ & \\
\hline & Negative & $106(90.6)$ & $31(56.4)$ & $27.053(0.000)$ \\
\hline RVFRV infection in Humans & Positive & $2(16.7)$ & $5(20.8)$ & \\
\hline & Negative & $10(83.3)$ & $19(79.2)$ & $0.089(1.000)$ \\
\hline
\end{tabular}

\section{Rvfv Rna Detection In Human, Goat, And Mosquito Samples}

Aedes spp and Culex spp were the dominant species among collected mosquitoes. However, Mansonia spp and Anopheles spp mosquitoes were also collected in smaller numbers. Our analyses were focused on Aedes spp, Culex, spp as the main documented vectors for RVFV. When virus detection was performed using polymerase chain reaction (RT-qPCR), goats exhibited the highest infection rate of $4.1 \%$, followed by humans (2.6\%). Aedes spp had a higher infection rate of $2.3 \%$ compared to that of Culex spp which was recorded to be $1.5 \%$.

Table 4

Comparison of mean $\lg \mathrm{M} / \lg \mathrm{G}$ anti-RVFV antibody concentrations between goats and humans

\begin{tabular}{|c|c|c|c|c|c|c|c|c|}
\hline \multirow{4}{*}{$\begin{array}{l}\text { RVF } \\
\text { HUMAN } \\
\text { - RVF } \\
\text { ANIMAL }\end{array}$} & \multicolumn{5}{|c|}{ Paired Differences } & \multirow[t]{3}{*}{$\mathrm{t}$} & \multirow[t]{3}{*}{ df } & \multirow{3}{*}{$\begin{array}{l}\mathrm{P} \text { - } \\
\text { value }\end{array}$} \\
\hline & \multirow{2}{*}{$\begin{array}{l}\text { Mean } \\
\text { (difference) }\end{array}$} & \multirow[t]{2}{*}{ SD } & \multirow{2}{*}{$\begin{array}{l}\text { SE of } \\
\text { Mean }\end{array}$} & \multicolumn{2}{|c|}{$95 \% \mathrm{Cl}$ of the Difference } & & & \\
\hline & & & & Lower & Upper & & & \\
\hline & 0.0363451 & 0.7346983 & 0.0691146 & -0.1005966 & 0.173287 & 0.526 & 112 & 0.600 \\
\hline
\end{tabular}

\section{Discussion}

The main aim of this study was to examine the degree of exposure to RVFV in goats and humans. This study also sought to isolate RVFV in humans, goats, and key RVFV vector mosquitoes; Aedes spp and Culex $s p p$. Results from this study show that, although there has been no RVF outbreak reported in Tanzania since 2006-2007, antibodies to RVFV and the virus has been detected in humans and goats in Lower Moshi area. Findings from this study indicate that $13.2 \%$ and $23.3 \%$ of tested humans and goats had circulating 
antibodies to RVFV, respectively. Our findings emphasize an active exposure to RVFV during IEPs as previously reported by some studies across geo-ecological zones of Tanzania [19, 25-28].

In this study, goats had higher exposure rates to RVFV compared to humans. Aedes spp, the major vector for RVFV, is known to have bimodal daily feeding behavior with both exophagic and exophilic behaviors[29], feeding on a wide range of mammalian hosts. Consequently, this behavior can be implicated as a key behavior in its role as a vector for many zoonotic infections. Despite its preference for human hosts [29], we report higher seropositivity in goats. The transmission of RVFV is not absolutely dependent on the presence of vector mosquitoes. Direct human contact with infected animal tissues has been reported as a significant factor for its transmission from animals to humans[30-32]. Not all of the human participants in this study were directly involved in activities that bring them into direct contact with infected tissues such as infected aborted fetuses and those working in slaughterhouses, which could partly explain the lower seropositivity to RFVFV in humans compared to goats.

In the current study, RVFV RNA was detected in humans, goats, and mosquitoes. Goats exhibited the highest infection rate of $4.1 \%$, followed by humans (2.6\%). Viral RNA was also detected in $2.3 \%$ and $1.5 \%$ of tested Aedes spp and Culex spp mosquito pools. This study was conducted to shed light on the maintenance mechanisms of RVFV by investigating both exposure and infection rates in mammalian and arthropod vectors. To our knowledge, this is the first study conducted in Tanzania to concomitantly report on RVFV diagnosis in humans, animals, and mosquitoes. Many of the previous studies that sought to understand the epidemiology of RVFV in Tanzania, either focused on sero-epidemiology or could not detect RVFV RNA in mammalian and arthropod vectors. Although the interactions of arboviruses and their vectors are complex and their epidemiology is poorly understood, our findings support the hypothesis that during IEPs, RVFV is likely maintained by localized low-level transmission between mosquito vectors and mammalian hosts without any noticeable clinical symptoms[15, 32-34]. Evidence for RVFV transmission during IEPs has previously been reported among humans, livestock, and wild animals in Tanzania and elsewhere [1-4, 6, 19, 24-28, 33, 35-38]. Although goats were more seropositive to RVFV compared to humans, paired comparison of mean anti-RVFV IgG/lgM concentrations revealed no difference that existed between humans and goats.

Some factors were significantly associated with seropositivity to RVFV in humans including male gender, living more than 4 persons in a household, being older than 50 years, not using an insecticide-treated bednet, and higher RVFV seropositivity in goats. RVFV seropositivity in Humans was consequently associated with seropositivity in goats. Males, especially in agropastoral communities seem to be more active outdoors for various subsistence activities including farming and grazing which bring them into frequent contact with RVFV susceptible or infected animals. This finding stresses the need for continued distribution, access, and usage of LLINs, especially among rural and agro-pastoral communities that are more prone to zoonotic diseases.

The study site is characterized by features that are supportive of vector mosquito breeding and intimate human-animal interaction. In the absence of reports on RVFV infection in areas nearby the study area, [19] the detection of antibodies to RVFV in humans and goats and detection of RVFV in humans, goats, and 
mosquitoes in the study area suggests the site to be a potential RVF hotspot. The dominant pastoral grazing system in the study area and surrounding areas is manifested as unlimited movements of livestock as a result of environmental degradation of the wetland due to overstocking and overgrazing increases chances for introducing the disease into new areas. The absence of clinical manifestations among livestock and humans in the study area, which could be a consequence of herd immunity, seems to have escaped the knowledge of the veterinary and public health authorities, raising concerns about the available local and national capacity for preparedness and response machinery against zoonotic infectious with potential to cause fatal epidemics. Thus, there exists a critical need for improved surveillance of RVF transmission through detection of RVFV activity among humans, livestock, and vector mosquitoes.

Since passive surveillance of RVF is challenging in the absence of clinical features among humans and livestock, active surveillance is recommended and, where resources may be limited, targeted surveillance in high-risk areas (hot spots) will help prevent future RVF outbreaks. It is critically important to relook the national contingency plans used in RVF surveillance and response to RVF outbreaks, bearing in mind that observed active transmission of the virus occurs in the absence of expected clinical manifestations that have been the traditional RVF pointers for a long time such as massive abortions in livestock.

\section{Conclusion}

Here, we present data that reveals the detection of anti-RVFV antibodies in humans and goats and isolation of RVFV from humans, goats, and mosquitoes in an area with the necessary features for mosquito breeding. Collected during a dry season of IEP, our data suggests the Lower Moshi area as a potential hotspot for RVF, posing the danger of being a source of RVFV to other areas. Strategies for effective active surveillance of RVF that involve the identification of RVF hotspots for targeted control are recommended.

\section{List Of Abbreviations}

RVF: Rift Valley Fever; RVFV: Rift Valley Fever Virus; IgG: Immunoglobulin G; IgM: Immunoglobulin M; IEP: Inter epidemic Period; cELISA: competitive ELISA;RT-qPCR: Real Time quantitative Polymerase Chain Reaction; RNA: Ribonucleic acid.

\section{Declarations}

\section{Ethics approval and consent to participate}

This study was conducted after the approval of the Kilimanjaro Christian MedicalUniversity College (KCMUCo) Research and Ethics Committee (Certificate \#2419). Permission to conduct the study was also obtained from Kilimanjaro Regional and District Administrative Secretaries, District Medical and Veterinary Officers, and local village and ward executive officers of respective villages. After an explanation of the study aims and procedures to the study communities through community sensitization meetings, individuals were asked to voluntarily agree to participate in the study. Written informed consent was obtained from all study participants aged 18 years and above by signing consent forms whereas parents and/or legal guardians of participants under 18 years and participants who could not read or write consented on behalf. All authors 
hereby declare that all procedures in this study were performed in accordance with the ethicalstandards laid down in the 1964 Declaration ofHelsinki.

\section{Consent for publication}

Not applicable

\section{Availability of Data and Materials}

The datasets used and/or analyzed during the current study are available from the corresponding author on reasonable request

\section{Competing interests}

The authors declare that they have no competing interests

\section{Funding}

This study was conducted with financial support from the International Center for GeneticEngineering and Biotechnology (ICGEB), Grant\#CRP/TZA18-04 to JOC and The East AfricanConsortium for Clinical Research (EACCR-2) grant\# EDCTP-RegNet2015-1104T through the KCRINID node to BTM. This support is highly acknowledged.

\section{Authors' Contributions}

JOC, RSM, BTM conceived the project, overall study implementation and wrote the manuscript.

MSK, RMB, PGH, SIM participated in data collection and made significant inputs in writing the manuscript. MSK, JOC and JMV analyzed and interpreted data

\section{Acknowledgements}

We acknowledge the logistical support provided by KCRI research administrators Ms. Elizabeth Kussaga, Ms. Tupokigwe Jana, Ms. Zuhura Lintu, and CPA Mr. Gilbert Shao. We also acknowledge the assistance of the Regional, District, and Field Executive Officers for providing permission to conduct this study. We thank all participants who consented to be part of this study.

\section{References}

1. Georges TM, Justin M, Victor M, Marie KJ, Mark R, Leopold MM. Seroprevalence and virus activity of Rift Valley fever in cattle in eastern region of the Democratic Republic of the Congo. Journal of veterinary medicine 2018; 2018.

2. Gudo ES, Pinto G, Weyer J, le Roux C, Mandlaze A, Jose ArF, et al. Serological evidence of Rift Valley fever virus among acute febrile patients in Southern Mozambique during and after the 2013 heavy rainfall and flooding: implication for the management of febrile illness. Virology journal 2016; 13(1):96. 
3. Nanyingi MO, Munyua P, Kiama SG, Muchemi GM, Thumbi SM, Bitek AO, et al. A systematic review of Rift Valley Fever epidemiology 1931-2014. Infection ecology \& epidemiology 2015; 5(1):28024.

4. Murithi RM, Munyua P, Ithondeka PM, Macharia JM, Hightower A, Luman ET, et al. Rift Valley fever in Kenya: history of epizootics and identification of vulnerable districts. Epidemiology \& Infection 2011; 139(3):372-380.

5. Gale P, Kelly L, Snary EL. Pathways for entry of livestock arboviruses into Great Britain: assessing the strength of evidence. Transboundary and Emerging Diseases 2015; 62(2):115-123.

6. Sanderson CE, Jori F, Moolla N, Paweska JT, Oumer N, Alexander KA. Silent Circulation of Rift Valley Fever in Humans, Botswana, 2013GÇô2014. Emerging infectious diseases 2020; 26(10):2453.

7. Fawzy M, Helmy YA. The one health approach is necessary for the control of Rift Valley fever infections in Egypt: A comprehensive review. Viruses 2019; 11(2):139.

8. Sindato C, Karimuribo E, Mboera LE. The epidemiology and socio-economic impact of Rift Valley fever in Tanzania: a review. Tanzania journal of health research 2011; 13(5).

9. Chengula AA, Mdegela RH, Kasanga CJ. Socio-economic impact of Rift Valley fever to pastoralists and agro-pastoralists in Arusha, Manyara, and Morogoro regions in Tanzania. Springerplus 2013; 2(1):549.

10. Bashir RSE, Hassan OA. A One Health perspective to identify environmental factors that affect Rift Valley fever transmission in Gezira state, Central Sudan. Tropical medicine and health 2019; 47(1):54.

11. Lorenzo G, Lopez-Gil E, Warimwe GM, Brun A. Understanding Rift Valley fever: contributions of animal models to disease characterization and control. Molecular Immunology 2015; 66(1):78-88.

12. Terasaki K, Makino S. Interplay between the virus and host in Rift Valley fever pathogenesis. Journal of innate immunity 2015; 7(5):450-458.

13. Sumaye RD, Geubbels E, Mbeyela E, Berkvens D. Inter-epidemic transmission of Rift Valley fever in livestock in the Kilombero River Valley, Tanzania: a cross-sectional survey. PLoS Negl Trop Dis 2013; 7(8):e2356.

14. Sumaye RD. Epidemiology of the inter-epidemic Rift Valley fever transmission of the Kilombero Valley, Tanzania. 2019.

15. Lumley S, Horton DL, Hernandez-Triana LL, Johnson N, Fooks AR, Hewson R. Rift Valley fever virus: strategies for maintenance, survival and vertical transmission in mosquitoes. Journal of General Virology 2017; 98(5):875-887.

16. Chamchod F, Cantrell RS, Cosner C, Hassan AN, Beier JC, Ruan S. A modeling approach to investigate epizootic outbreaks and enzootic maintenance of Rift Valley fever virus. Bulletin of mathematical biology 2014; 76(8):2052-2072.

17. Pedro SA, Abelman S, Tonnang HE. Predicting rift valley fever inter-epidemic activities and outbreak patterns: Insights from a stochastic host-vector model. PLoS neglected tropical diseases 2016; 10(12):e0005167.

18. Breiman RF, Njenga MK, Cleaveland S, Sharif SK, Mbabu M, King L. Lessons from the 2006 GÇô2007 Rift Valley fever outbreak in East Africa: implications for prevention of emerging infectious diseases. 2008.

19. Nyarobi MJ. The epidemiology of Rift Valley fever in northern Tanzania. 2020. 
20. Lowassa A, Mazigo HD, Mahande AM, Mwang'onde BJ, Msangi S, Mahande MJ et al.,. Social economic factors and malaria transmission in Lower Moshi, northern Tanzania. Parasites \& vectors. 2012 Dec;5(1):1-9.

21. Bell JA, Mickelson NJ, Vaughan JA. West Nile virus in host-seeking mosquitoes within a residential neighborhood in Grand Forks, North Dakota. Vector-Borne \& Zoonotic Diseases 2005; 5(4):373-382.

22. aciel-de-Freitas, R., Eiras, Á.E. and Lourenço-de-Oliveira. Field evaluation of effectiveness of the BGSentinel, a new trap for capturing adult Aedes aegypti (Diptera: Culicidae). Memórias do Instituto Oswaldo Cruz 2006; 101(3):321-325.

23. Wensman JJ, Lindahl J, Wachtmeister N, Torsson E, Gwakisa P, Kasanga C, et al. A study of Rift Valley fever virus in Morogoro and Arusha regions of Tanzania: serology and farmers' perceptions. Infection ecology \& epidemiology 2015; 5(1):30025.

24. Budodo RM, Horumpende PG, Mkumbaye SI, Mmbaga BT, Mwakapuja RS, Chilongola J. Serological evidence of exposure to Rift Valley, Dengue and Chikungunya Viruses among agropastoral communities in Manyara and Morogoro regions in Tanzania: A community Survey. 2020.

25. Ahmed A, Makame J, Robert F, Julius K, Mecky M. Sero-prevalence and spatial distribution of Rift Valley fever infection among agro-pastoral and pastoral communities during Interepidemic period in the Serengeti ecosystem, northern Tanzania. BMC infectious diseases 2018; 18(1):276.

26. Chengula AA, Kasanga CJ, Mdegela RH, Sallu R, Yongolo M. Molecular detection of Rift Valley fever virus in serum samples from selected areas of Tanzania. Tropical animal health and production 2014; 46(4):629-634.

27. Heinrich N, Saathoff E, Weller N, Clowes P, Kroidl I, Ntinginya E, et al. High seroprevalence of Rift Valley fever and evidence for endemic circulation in Mbeya region, Tanzania, in a cross-sectional study. PloS Negl Trop Dis 2012; 6(3):e1557.

28. Matiko MK, Salekwa LP, Kasanga CJ, Kimera SI, Evander M, Nyangi WP. Serological evidence of interepizootic/inter-epidemic circulation of Rift Valley fever virus in domestic cattle in Kyela and Morogoro, Tanzania. PLoS neglected tropical diseases 2018; 12(11):e0006931.

29. Delatte H, Desvars A, Bou+ᄀtard A, Bord S+, Gimonneau G, Vourc'h Gl, et al. Blood-feeding behavior of Aedes albopictus, a vector of Chikungunya on La R+ᄀunion. Vector-Borne and Zoonotic Diseases 2010; 10(3):249-258.

30. Nyakarahuka L, Maurice AdS, Purpura L, Ervin E, Balinandi S, Tumusiime A, et al. Prevalence and risk factors of Rift Valley fever in humans and animals from Kabale district in Southwestern Uganda, 2016. PLoS neglected tropical diseases 2018; 12(5):e0006412.

31. Olovsson E. Seroprevalence and risk factors for Rift Valley fever and Capripoxvirus in small ruminants in the border region of Tanzania-Zambia. 2019.

32. Nyarobi MJ. The epidemiology of Rift Valley fever in northern Tanzania. 2020.

33. Salekwa LP, Wambura PN, Matiko MK, Watts DM. Circulation of Rift Valley Fever Virus Antibody in Cattle during Inter-Epizootic/Epidemic Periods in Selected Regions of Tanzania. The American journal of tropical medicine and hygiene 2019; 101(2):459-466. 
34. Stoek AF. Maintenance of Rift Valley fever phlebovirus during inter-epidemic periodsGÇôMonitoring of vectors and identification of potential reservoir hosts. 2020.

35. LaBeaud ADe, Muiruri S, Sutherland LJ, Dahir S, Gildengorin G, Morrill J, et al. Postepidemic analysis of Rift Valley fever virus transmission in northeastern Kenya: a village cohort study. PLoS neglected tropical diseases 2011; 5(8).

36. Lumley S, Horton DL, Hernandez-Triana LL, Johnson N, Fooks AR, Hewson R. Rift Valley fever virus: strategies for maintenance, survival and vertical transmission in mosquitoes. Journal of General Virology 2017; 98(5):875-887.

37. Nanyingi MO, Muchemi GM, Thumbi SM, Ade F, Onyango CO, Kiama SG, et al. Seroepidemiological survey of Rift Valley fever virus in ruminants in Garissa, Kenya. Vector-Borne and Zoonotic Diseases 2017; 17(2):141-146.

38. Swai ES, Schoonman L. Prevalence of Rift Valley fever immunoglobulin $\mathrm{G}$ antibody in various occupational groups before the 2007 outbreak in Tanzania. Vector-Borne and Zoonotic Diseases 2009; 9(6):579-582.

\section{Figures}




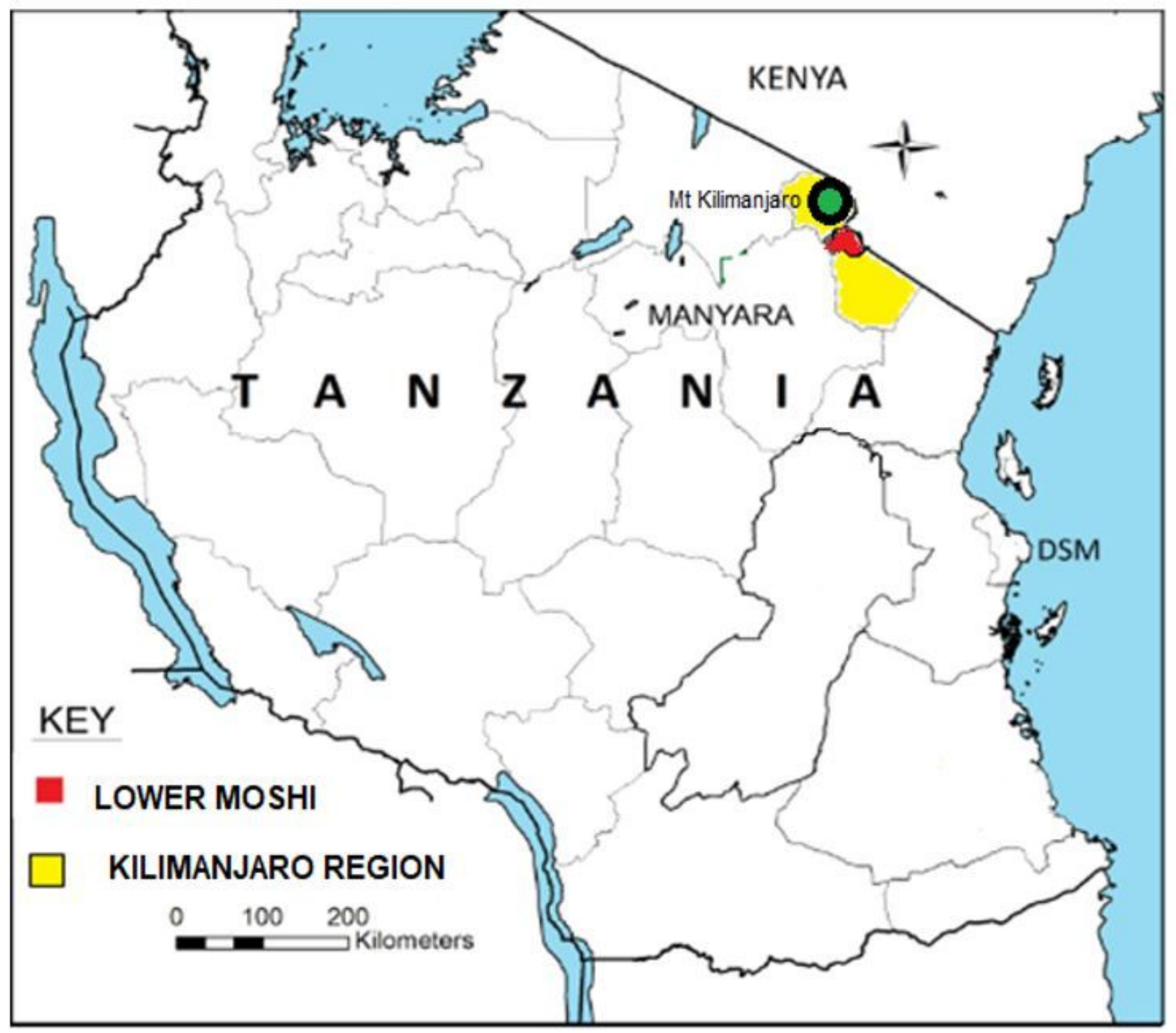

Figure 1

Map of Tanzania developed from Google earth showing study site. Note: The designations employed and the presentation of the material on this map do not imply the expression of any opinion whatsoever on the part of Research Square concerning the legal status of any country, territory, city or area or of its authorities, or concerning the delimitation of its frontiers or boundaries. This map has been provided by the authors. 


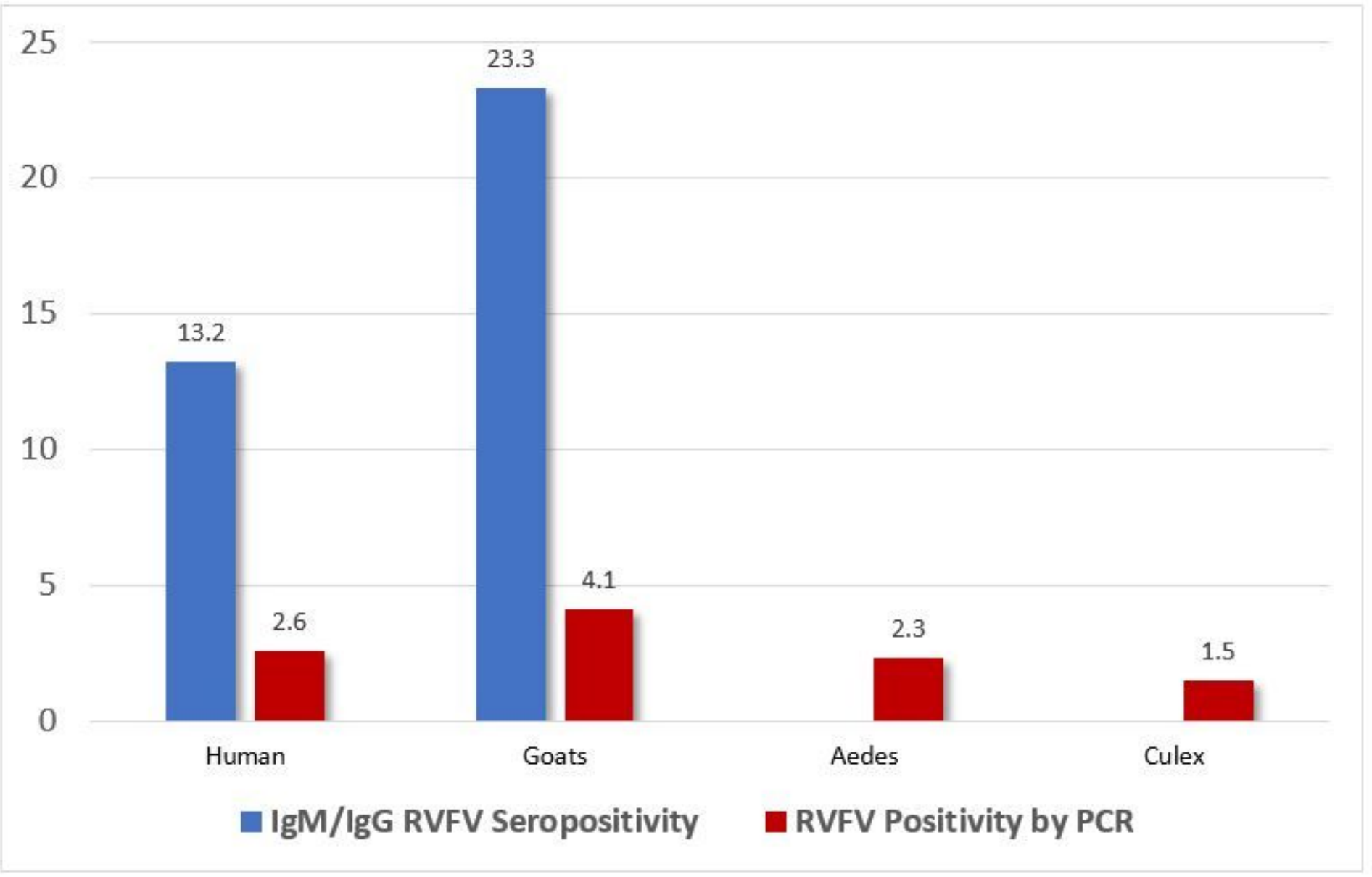

Figure 2

Comparative $\lg \mathrm{M} / \lg \mathrm{G}$ seropositivity and PCR positivity between goats, mosquitoes and humans 